\title{
Laser polishing and 2PP structuring of inside microfluidic channels in fused silica
}

\author{
Christian Weingarten ${ }^{1}$ (D) $\cdot$ Sönke Steenhusen ${ }^{2} \cdot$ Martin Hermans $^{3}$. \\ Edgar Willenborg ${ }^{1} \cdot$ Johannes Henrich Schleifenbaum $^{1}$
}

Received: 13 March 2017 / Accepted: 22 September 2017 / Published online: 20 October 2017

(c) The Author(s) 2017. This article is an open access publication

\begin{abstract}
This study presents the development of postprocessing steps for microfluidics fabricated with selective laser etching (SLE) in fused silica. In a first step, the SLE surface-even inner walls of microfluidic channels—can be smoothed by laser polishing. In addition, two-photon polymerization (2PP) can be used to manufacture polymer microstructures and microcomponents inside the microfluidic channels. The reduction in the surface roughness by laser polishing is a remelting process. While heating the glass surface above softening temperature, laser radiation relocates material thanks to the surface tension. With laser polishing, the RMS roughness of SLE surfaces can be reduced from $12 \mu \mathrm{m}$ down to $3 \mathrm{~nm}$ for spatial wavelength $\lambda<400 \mu \mathrm{m}$. Thanks to the laser polishing, fluidic processes as well as particles in microchannels can be observed with microscopy. A manufactured microfluidic demonstrates that SLE and laser polishing can be combined successfully. By developing two-photon polymerization (2PP) processing in microchannels we aim to enable new applications with sophisticated $3 \mathrm{D}$ structures inside the microchannel. With $2 \mathrm{PP}$, lenses with a diameter of $50 \mu \mathrm{m}$ are processed with a form accuracy rms of $70 \mathrm{~nm}$. In addition, this study demonstrates that 3D structures can be fabricated inside the microchannels manufactured with SLE. Thanks to the combination of SLE, laser polishing and 2PP, research is pioneering new applications for microfluidics made of fused silica.
\end{abstract}

Christian Weingarten

christian.weingarten@ilt.fraunhofer.de

1 Fraunhofer Institute for Laser Technology (ILT), Steinbachstr 15, 52074 Aachen, Germany

2 Fraunhofer Institute for Silicate Research (ISC), Neunerplatz 2, 97082 Würzburg, Germany

3 LightFab GmbH, Steinbachstr 15, 52074 Aachen, Germany
Keywords Microchannel $\cdot$ Microfluidic $\cdot$ Laser polishing $\cdot 2 \mathrm{PP} \cdot$ Selective laser etching $\cdot$ SLE

\section{Introduction}

The fabrication and functionalization of microfluidic chips has attracted much interest in recent years. Whereas softlithography and molding in PDMS appear to be the standard for lab-scale demonstrations, other technologies such as 3D printing using stereolithography or inkjet and subtractive manufacturing (e.g., ablation) are also available options (Waheed et al. 2016). The selective laser etching process (SLE) enables microfluidics to be fabricated out of fused silica. Indeed, SLE makes it possible to machine hollow volumes into transparent materials with a huge freedom of geometry in three dimensions.

SLE is a two-step process used for microstructuring of glass materials. In the first step, ultrashort pulsed laser radiation is tightly focused into the inside of transparent material. When the intensity exceeds the threshold for nonlinear absorption in the vicinity of the focal point, laser radiation is absorbed and the material becomes locally heated to several thousand degrees Celsius (Hermans et al. 2012). Since heated volume is small, extreme cooling rates occur and the material is frozen in the highly excited state. Line-by-line and layer-by-layer, a complete 3D connected grid of lines can be permanently inscribed into the bulk material.

Subsequently, the modified material can be selectively removed in a second process step by wet-chemical etching, making use of the fact that the modified material shows an increased etching rate for the etching liquid used. The ratio of etching rates between modified and unmodified material is called selectivity and can be understood as an upper limit of aspect ratios that can be achieved. For fused silica, the 
maximum selectivity was found to be $\sim 1000$ for etching with an aqueous solution of $\mathrm{KOH}$ (Hermans 2014). In addition to fused silica, the SLE process has been demonstrated in photoreactive Foturan glass (Sugioka and Cheng 2014). Much earlier, Sugioka first demonstrated fs-laser-induced chemical etching of Foturan (Cheng et al. 2003). In comparison with the SLE of fused silica, the Foturan structuring has some disadvantages. As an additive in glass, for example, silver can have severe impact on cells in microfluidic applications. Furthermore, the selectivity found for the structuring of Foturan is lower than the one achieved with SLE in fused silica (Sima et al. 2015). This induces low structural accuracy, e.g., by the presence of conical channels.

The resulting surfaces of the SLE process exhibit high roughness, a disadvantage that impedes microscopic imaging and potentially further manufacturing (2PP) through the glass. This can partially be circumvented by an annealing step that smooths the walls due to surface tension (Wu et al. 2014). He et al. (2011) fabricated hollow optical waveguides in fused silica by femtosecond laser micromachining and reduced the roughness afterward by a post-annealing process. Thanks to the post-annealing (annealing temperature: $1200{ }^{\circ} \mathrm{C}$ ) the surface roughness Ra could be reduced from 101.5 to $4.6 \mathrm{~nm}$ (measuring field $10 \times 10 \mu \mathrm{m}^{2}$ ). One advantage of the post-annealing is that all inner surfaces are smoothed. Another option is to provide an area of low surface roughness by fabricating a thin scaffold layer with $2 \mathrm{PP}$ (Wu et al. 2015). However, global annealing might affect the cross-sectional area of very fine 3D channels; moreover, providing $2 \mathrm{PP}$-written layer is time consuming and limited to small areas. For this reason, laser polishing can be used to reduce the roughness of SLE-written structures without heating the entire substrate. Jung et al. (2016) and Serhatlioglu et al. (2016) tested polishing of open microchannels with $\mathrm{CO}_{2}$ laser radiation. Both could reduce the roughness of the microchannel down to approx. $60 \mathrm{~nm}$.

Laser polishing is a process that remelts the surface without almost any material loss. Since the laser radiation is absorbed in a thin surface layer, the glass is heated up to $50-100{ }^{\circ} \mathrm{C}$ below evaporation temperature. When the surface is heated to this degree, the viscosity decreases as does the roughness because the material is relocated on account of the surface tension. In recent years, laser polishing of glass with $\mathrm{CO}_{2}$ laser radiation has been developed for the manufacturing of optics (Weingarten et al. 2017; Heidrich et al. 2015; Hildebrand et al. 2011; Nowak et al. 2006), scratch removal of large fused silica optics (Cormont et al. 2013) as well as for the processing of optical fibers (Heptonstall 2014).

To enhance the functionality of microfluidic systems by $3 \mathrm{D}$ microstructures, direct-laser writing or two-photon polymerization (2PP) has been widely recognized as a valuable technology. 2PP relies on strongly confined solidification of a photopolymer triggered by two-photon absorption inside the focal volume of femtosecond laser pulses. Scanning the focal volume in 3D together with a subsequent development step results in arbitrary microstructures (Houbertz et al. 2010). In recent years, $2 \mathrm{PP}$ has been used to manufacture structures such as micropumps, valves, mixers and micromotors inside fluidic channels (Eaton et al. 2012; Lin et al. 2015; Sugioka and Cheng 2014). In addition, more sophisticated materials than polymers-enzymes (Iosin et al. 2011) and silver containing composites (Focsan et al. 2016) — could be assembled on microfluidic scales. However, polydimethylsiloxane (PDMS), which is the standard material for microfluidic cells, is hydrophobic, permeable to water vapor and difficult to process by photopatterning. Poly (methyl methacrylate) (PMMA), on the other hand, could be an option in which microfluidic channels can be written by laser ablation. However, these channels have to be sealed after manufacturing by, e.g., a cover glass (Eaton et al. 2015). The same applies to a combined approach with $2 \mathrm{PP}$ as an additive process and ablation in the same photopolymer (Xiong et al. 2012). Consequently, demand has grown for enclosed 3D microfluidic systems inside glass (Amato et al. 2012; Wu et al. 2014, 2015). Selective laser etching (SLE) has proven to be an ideal process to fabricate such systems.

In this paper we demonstrate the results for the combination of SLE, laser polishing and 2PP for the fabrication of microfluidic devices. It is particularly attractive that SLE and 2PP manufacturing can be conducted on the same patterning hardware.

\section{Experimental setup}

\subsection{Selective laser etching (SLE)}

The SLE parts for testing of laser polishing and 2PP were produced with a LightFab 3D Printer: a CAD model was sliced in layers with a variable distance in the $\mathrm{z}$ direction (down to $100 \mathrm{~nm}$ depending on the curvature) in order to derive the data for the tool path the laser spot has to move along. Additional coarse gridlines were added to speed up etching. After all the modification lines were irradiated, the sample was etched for $48 \mathrm{~h}$ in a heated bath of $\mathrm{KOH}$. Fused silica was used to test the combination of SLE and laser polishing.

\subsection{Laser polishing}

For laser polishing, $\mathrm{CO}_{2}$ laser radiation with a wavelength of $\lambda=10.6 \mu \mathrm{m}$ is used because fused silica has a low optical penetration depth of 4-30 $\mu \mathrm{m}$ (depending on the temperature) (McLachlan and Meyer 1987) and absorbs nearly 80\% 


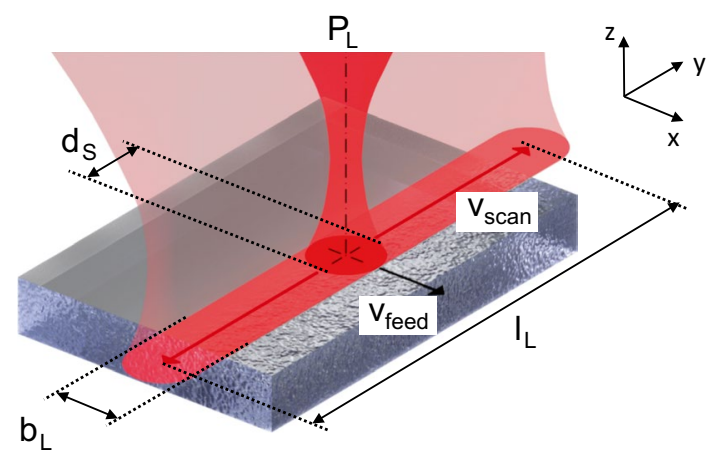

Fig. 1 Procedural principle of laser polishing of glass material. $P_{\mathrm{L}}=$ laser power, $v_{\text {feed }}=$ feed speed, $v_{\text {scan }}=$ scan speed, $b_{\mathrm{L}}$, $l_{\mathrm{L}}=$ width, length of the quasi-line, $d_{\mathrm{S}}=$ laser beam diameter (Weingarten et al. 2017)

of the radiation. Hence, a thin surface layer of the glass can be heated up to temperatures above softening temperature of glass.

The procedural principle of laser polishing is shown in Fig. 1. The defocused laser beam $\left(d_{\mathrm{S}}=5-10 \mathrm{~mm}\right)$, controlled by a galvanometric scanner, is moved across the surface at scan speed $v_{\text {scan }}$ up to $10 \mathrm{~m} / \mathrm{s}$ in the y direction. In addition, the laser scanner moves in the $x$ direction at feed speed $v_{\text {feed }}$ in the range of $1-5 \mathrm{~mm} / \mathrm{s}$. Since the laser beam moves faster than the feed speed, the scan tracks can be seen as quasi-lines. In the center of the quasi-line, the surface temperature is measured for a PID control to achieve a homogeneous temperature distribution during the process. The temperature control prevents the material from heating above evaporation temperature. To measure the temperature, we used a pyrometer DT10G made by DIAS Infrared $\mathrm{GmbH}$ with a spectral range around $5 \mu \mathrm{m}$. Due to the infrared spectral range, the surface temperature is measured with the pyrometer (measuring depth: approx. $100 \mu \mathrm{m}$ ).

Figure 2 shows the experimental setup for laser polishing. Remaining dust particles can lead to surface defects, while the surface layer is melted. To prevent dust particles from depositing on the glass sample, the experimental setup is placed in a flow box. $\mathrm{A} \mathrm{CO}_{2}$ laser with max. power of $1.5 \mathrm{~kW}$ and a Gaussian intensity profile is used.

A white-light interferometer from Zygo-Ametek $\mathrm{GmbH}$ is used for the surface measurements. The vertical resolution of the measurement system is $<1 \mathrm{~nm}$ and the lateral resolution $>110 \mathrm{~nm}$.

\subsection{Two-photon polymerization (2PP)}

For practical applications, 2PP and SLE fabrication can be conducted on the same hardware as both processes are triggered using $515 \mathrm{~nm}$ femtosecond laser pulses. However, the work presented here was carried out on a machine

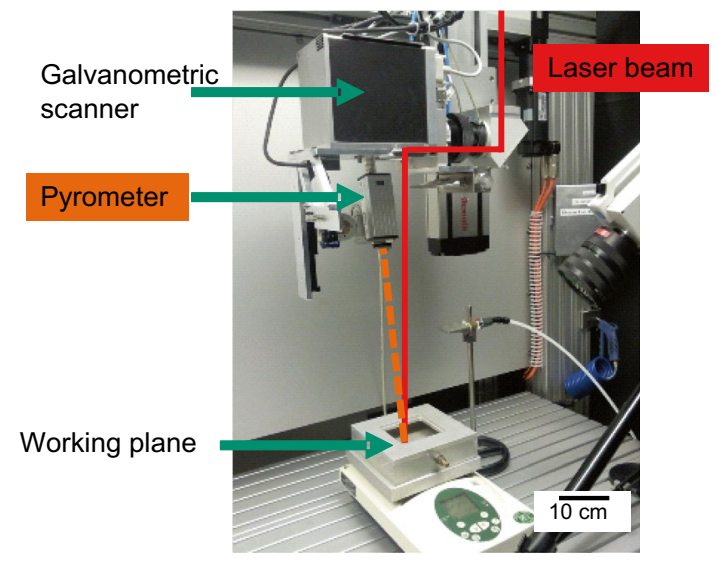

Fig. 2 Experimental setup for laser polishing of glass

designated only for $2 \mathrm{PP}$. This machine consists of a femtosecond oscillator (Amplitude Systems t-pulse 200) emitting $1030 \mathrm{~nm}$ pulses at a repetition rate of $10 \mathrm{MHz}$. The 350-fs pulses are coupled into an acousto-optical modulator for shuttering on a $\mu$ s scale and finally frequency doubled to $515 \mathrm{~nm}$. Before the laser pulses are focused, the beam is expanded with a $3 \times$ telescope and spatially filtered with a combination of focusing lens, pinhole and collimation lens. The laser power, or rather the pulse energy, can be adjusted by combing computer-controlled $\lambda / 2$-plate and a polarizing beam splitter.

Independent positioning systems place the focal spot in the desired location. For axial motion, a piezo stage (Piezosystem Jena) and a long travel linear stage (Aerotech ATS) were used. In the lateral plane, the sample can be positioned with high precision air bearing linear stages (Aerotech ABL) that are used for stitching and coarse positioning of the substrate. These can be employed directly for the hatching of the desired structure and are particularly beneficial for structures larger than the field of view of the focusing optics employed (Stichel et al. 2010). However, in the experiments presented here, the hatching was conducted by galvanometric mirrors (LightFab GmbH; Scanlab) to significantly increase the throughput. A schematic illustration of the structuring setup can be found elsewhere (Houbertz et al. 2010; Steenhusen et al. 2010).

OC-I, an ORMOCER ${ }^{\circledR}$ hybrid polymer developed by Fraunhofer ISC, was used as a photoresist (Fessel et al. 2012; Sanchez et al. 2011). OC-I was chosen because it is compatible to the $2 \mathrm{PP}$ processing and is biocompatible (Burmeister et al. 2015; Doraiswamy et al. 2005). Consequently, OC-I is a promising photoresist for applications in microfluidic and lab-on-chip devices. In case of samples outside of microfluidic channels, a drop of OC-I was applied onto a standard microscope cover slip (Schott D263 ${ }^{\circledR} \mathrm{M}$ ) as substrate. Structures were fabricated on the backside of this substrate using microscope objectives with numeric 
apertures (NA) of 0.6 and 1.4 (oil-immersion), respectively. Samples were developed for $10 \mathrm{~min}$ in MIBK:isopropanol (1:1) and finally rinsed with pure isopropanol. The structures inside microfluidic channels were infused with OC-I using a syringe. Development was conducted with the same chemicals as above. These were pressed into the channels several times in order to flush away the remaining liquid polymer.

\section{Results}

\subsection{Laser polishing}

The microroughness of the SLE surface is dominated by the etching process. As seen in the white-light interferometer images in Fig. 3, spherical craters with a typical diameter of $10 \mu \mathrm{m}$ occur during the etching process. The waviness of the SLE surface is influenced by the irregular structuring in the scan field $\left(A_{\mathrm{sf}} \approx 300 \times 300 \mu \mathrm{m}^{2}\right)$. A systematical error of the lenses causes a spherical convex form of each scan field on the surface. Hence, when a flat surface is processed with SLE, the center of each scan field is lower than the edge of the scan field with a PV of approx. $3 \mu \mathrm{m}$.

To test laser polishing, a $10 \times 10 \mathrm{~mm}^{2}$ flat surface was processed with SLE and afterward laser polished at feed speed $v_{\text {feed }}=1 \mathrm{~mm} / \mathrm{s}$ and laser beam diameter $d_{\mathrm{S}}=7 \mathrm{~mm}$. The surface was measured before and after laser polishing with the white-light interferometer in three magnifications. The images of the SLE surface and after laser polishing are shown in Fig. 3. To analyze the roughness quantitatively, we plotted it as a function of the spatial wavelength in a roughness diagram (Fig. 4).

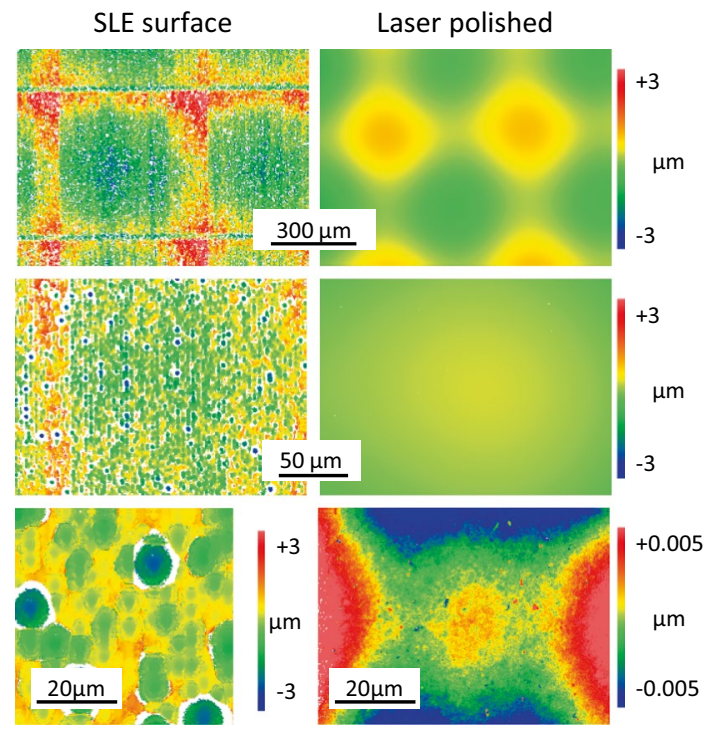

Fig. 3 White-light interferometer images with different magnifications of the SLE (left) and laser polished (right) surface

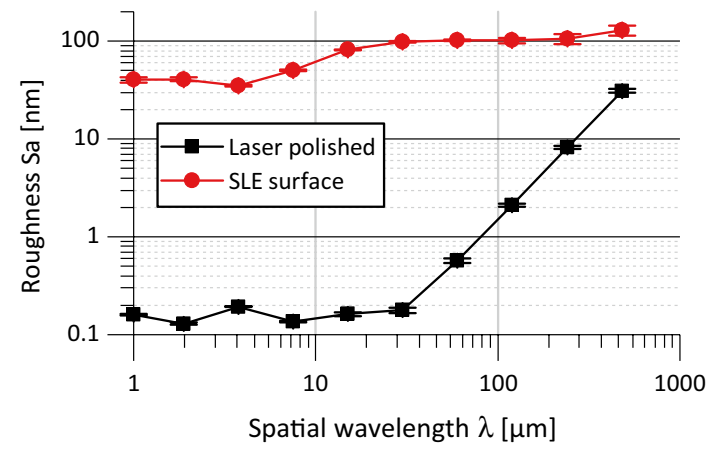

Fig. 4 Roughness $\mathrm{Sa}$ in dependence on the spatial wavelength for SLE and laser polished surfaces

With laser polishing, the microroughness of the SLE surface can be reduced significantly. Up to spatial wavelength $\lambda=30 \mu \mathrm{m}$, the roughness Sa can be reduced from 50 to $100 \mathrm{~nm}$ down to $0.2 \mathrm{~nm}$. This microroughness is already sufficient for imaging optics. The periodic surface structure due to the scan field inhomogeneity (waviness) can be also reduced, but a residual roughness $\mathrm{Sa}>10 \mathrm{~nm}$ remains on the surface. To further reduce the waviness, another laser-based process step (laser beam figuring) can be added to selectively ablate material. A detailed description of laser beam figuring can be found in Weingarten et al. (2015).

In addition, due to the thermal process, laser polishing can be used to smooth internal surfaces in the bulk material. The thermal penetration $l_{\text {th }}$ during the interaction of laser radiation and the glass surface can be described by

$l_{\mathrm{th}}=2 \cdot \sqrt{k \cdot t}$,

where $t$ is the interaction time and $k$ the thermal diffusivity. In this paper, the interaction time $t$ is approximately described by

$t=\frac{b_{\mathrm{L}}}{v_{\text {feed }}}=\frac{d_{\mathrm{S}}}{v_{\text {feed }}}$

with the width of the quasi-line $b_{\mathrm{L}}$, the laser beam diameter $\mathrm{d}_{\mathrm{S}}$ and the feed speed $v_{\text {feed }}$. During laser polishing of fused silica $\left[k=0.842 \times 10^{-6} \mathrm{~m}^{2} / \mathrm{s}\right.$ (Fanderlik 1991)], the thermal penetration depth is deeper by a factor $10^{3}$ than the optical penetration depth. If the wall thickness of the laser polished surface is thinner than the penetration depth of the softening temperature isotherm, the inner surface is smoothed as material relocates. A sketch of the procedural principle is shown in Fig. 5. The temperature control with a pyrometer prevents the upper surface from heating above the evaporation temperature. Due to the small wall thickness and, hence, the reduced heat conduction into the bulk material, even 


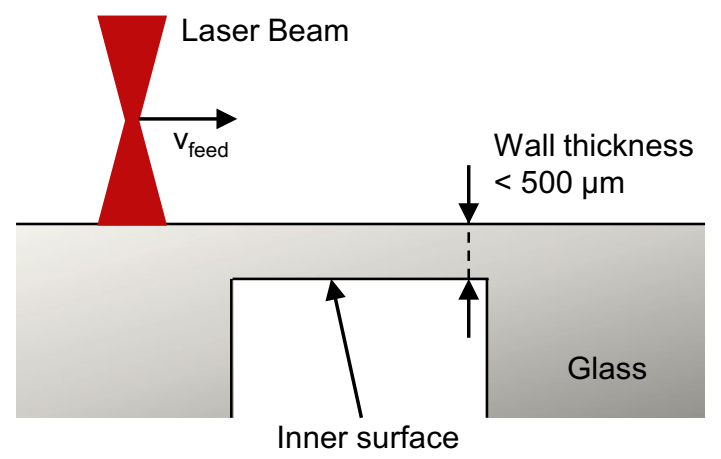

Fig. 5 Procedural principle of inside laser polishing of microchannels

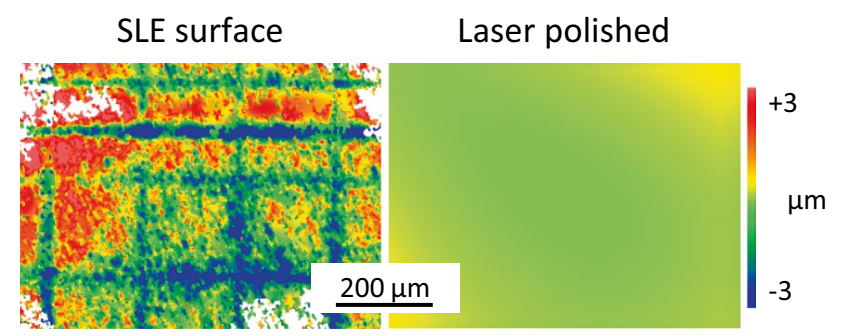

Fig. 6 White-light interferometer images of the SLE and laser polished inside surface

less interaction time is necessary to heat up the surface to process temperature.

Laser polishing of inner walls was tested on windows with a dimension of 5 by $5 \mathrm{~mm}^{2}$ and a wall thickness of $500 \mu \mathrm{m}$ manufactured with SLE (Fig. 5). The white-light images of the inner surface after SLE processing and laser polishing are shown in Fig. 6. The microroughness of the inner surface (high pass filter: $\lambda=400 \mu \mathrm{m}$ ) in a measurement field of 1 by $1 \mathrm{~mm}^{2}$ is reduced from 1.2 to $3 \mathrm{~nm}$.

So that the visual quality of the inside polished surface could be tested for microscopy, microchannels were fabricated with SLE and afterward laser polished. The height of the microchannels is $300 \mu \mathrm{m}$, the width $800 \mu \mathrm{m}$ and the wall thickness $300 \mu \mathrm{m}$. Figure 7 shows the microscopic images of a human hair inside the microchannels through the initial SLE surface and the laser polished surface. Since the SLE surface has such a high microroughness, it is not possible to obtain sharp microscopic images of the hair as well as of the edges of the microchannel. After laser polishing, the surface is smoothed and hence a sharp microscopy image of the hair can be taken.

A microfluidic device was designed to demonstrate that the combination of SLE and laser polishing is feasible. Every surface of the demonstrator is processed by SLE. The wall thickness between the outer surface and

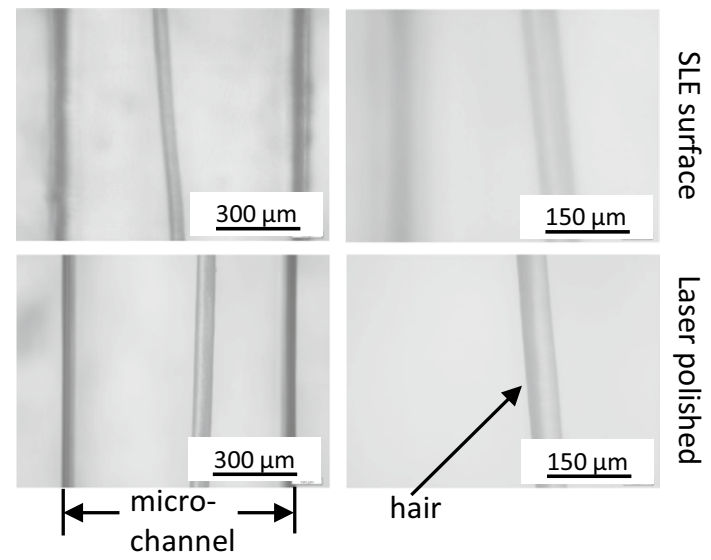

Fig. 7 Microscopy images of a hair in microchannels processed with SLE (above) and laser polished (below)
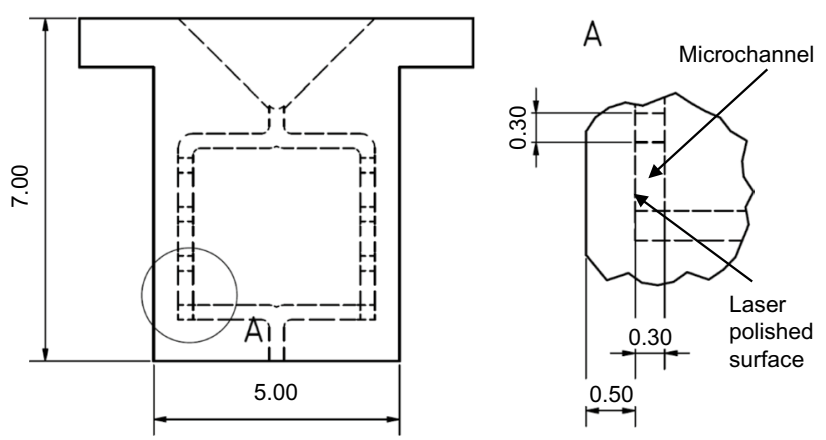

Fig. 8 Technical drawing of the demonstrator. Dimensions in $\mathrm{mm}$

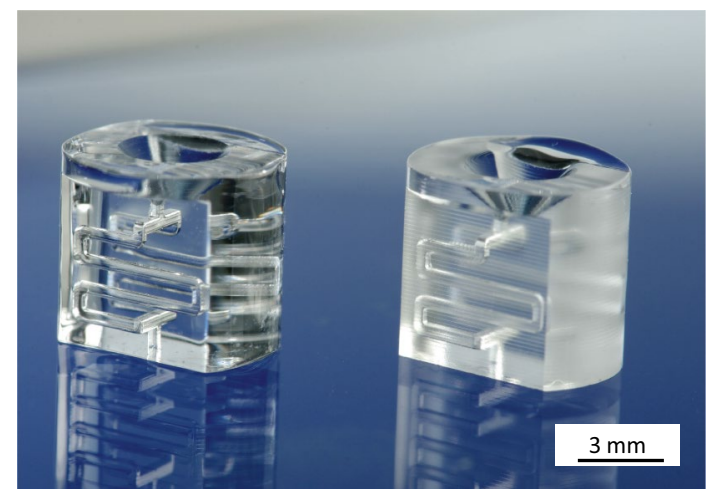

Fig. 9 Demonstrator for laser polished SLE parts. Right: after SLE process; left: laser polished

the microchannel is $500 \mu \mathrm{m}$ and the width of the channel $300 \mu \mathrm{m}$. A sketch of the demonstrator is shown in Fig. 8.

In Fig. 9, the demonstrator is shown after manufacturing with SLE and after laser polishing. The roughness of the outer walls of the demonstrator is similar to the results 


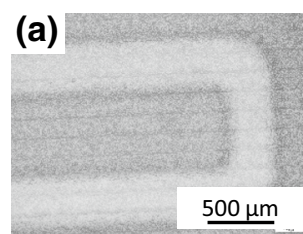

(c)
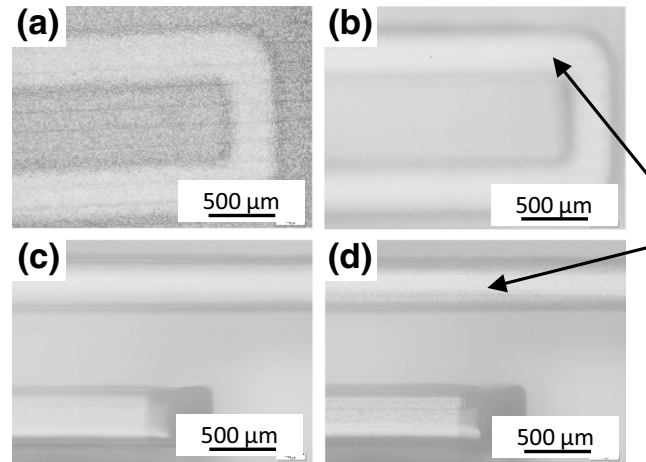

(d)
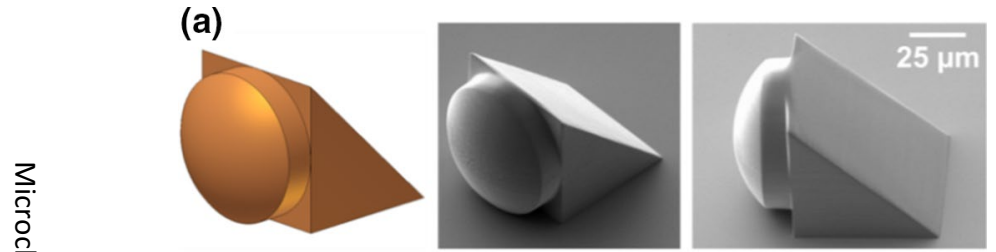

(b)
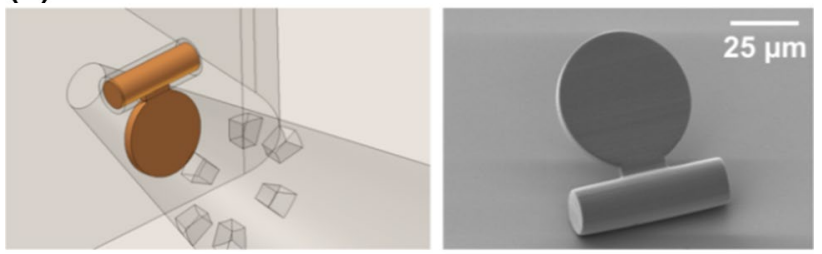

(c) tor. a Surface after SLE, b laser polished surface, $\mathbf{c}$ focused on the inner surface of the channel, $\mathbf{d}$ focused on the ground surface of the channel

shown in Fig. 4. In addition it can be seen that the inner walls can also be smoothed with laser polishing. Due to the small dimension of the microchannels inside the bulk material, white-light interferometer cannot be used to measure the inside surfaces. Nonetheless, the microscopy images of the demonstrator show that with laser polishing the roughness of the outer surface (Fig. 10b) as well as the inner surface of the microchannel (Fig. 10c) is reduced compared to the initial SLE surface (Fig. 10a). Compared to the post-annealingall inner surfaces are smoothed-with laser polishing the ground surface of the microchannel is not affected. Hence, the initial SLE surface of the ground surface can be seen in the microscopic images (Fig. 10d). Due to the geometry of the demonstrator, the roughness of the side walls could not be measured. This setup demonstrates that microfluidics in fused silica can be processed with SLE and laser polishing for visual observation of the fluidic processes by microscopy.

\subsection{PP}

As this paper mainly aims to demonstrate the potential of combining SLE, 2PP and laser polishing, different polymer structures were defined which might complement the functionality of SLE fabricated channels in fused silica. These structures comprise microlenses and total internal reflection mirrors (TIR mirror) for optofluidic applications, flaps for directing fluidic flow and finally a tiny microfluidic redistributor inside fused silica channels. Renderings of these structures are shown in Fig. 11 together with preliminary SEM characterization. In that case they were fabricated using the NA $=1.4$ microscope objective.

As can clearly be seen, with appropriate writing conditions, the target design can be reproduced. The structures exhibit very distinct edges, and the flap is perfectly aligned to the substrate at an angle of $45^{\circ}$. Each of the depicted structures was also fabricated using the lower NA $(\mathrm{NA}=0.6)$

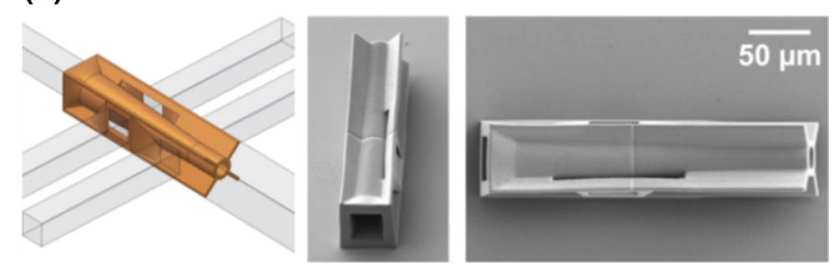

Fig. 11 Design and SEM characterization of microfluidic polymer structures

optics. These experiments are not discussed here in detail, but resulted in equivalent findings. In contrast to $\mathrm{NA}=1.4$, it was possible to scale the overall dimensions by a factor of approx. four, without a significant increase in the overall fabrication time.

In the case of the TIR mirror and the flaps, the fabrication time is in the order of 5-20 min, while the more complex microfluidic redistributor takes about $1-2 \mathrm{~h}$. The similar fabrication time of low-NA and high-NA optics is due to faster positioning velocities and larger volume pixels (voxel) for low-NA optics. The latter allows larger slicing and hatching distances without influencing the quality and stability of the structures. On the other hand, employing low-NA optics leads to higher dose sensitivity and particularly decreased accuracy in areas with small lateral cross sections such as the tip of the TIR mirror or the top of an individual lens. This effect is depicted in Fig. 12, showing the dose dependence for a microlens (Fig. 12a-c) and affected precision of a TIR mirror (Fig. 12d).

Even with the NA $=1.4$ objective, the fabricated surface still deteriorates, potentially owing to polymer shrinkage and inhomogeneous polymer-cross-linking. Another factor that influences the surface quality is the slicing and hatching procedure, which results in noticeable contour lines on the surface. Figure 13 depicts further characterization of the TIR mirror (a) and a microlens (b).

On first glance, the topography of the TIR mirror characterized by laser scanning microscopy (LSM, lateral resolution $>100 \mathrm{~nm}$, vertical resolution $>0.5 \mathrm{~nm}$ ) 

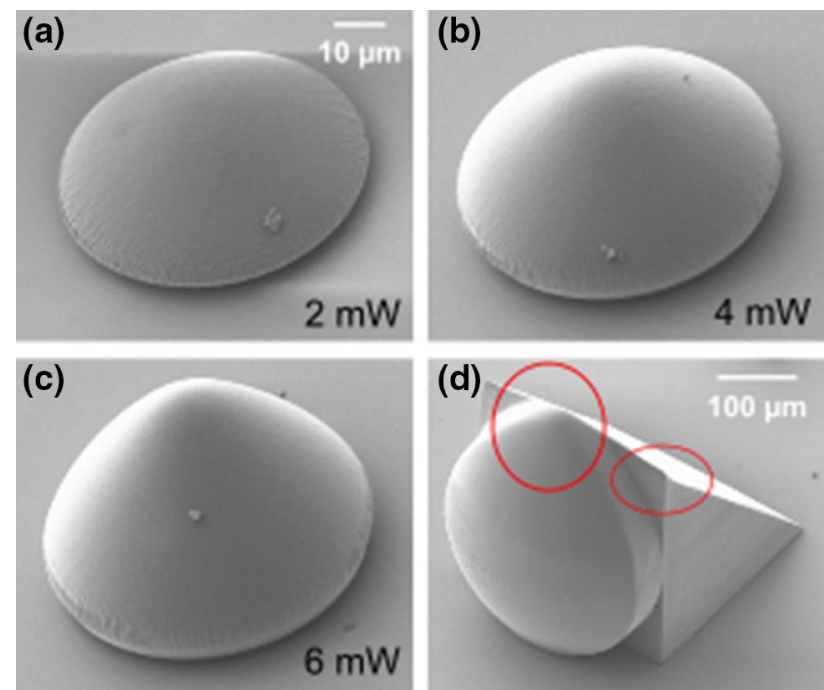

Fig. 12 SEM characterization of microlenses with different exposure doses and a TIR mirror revealing high dose sensitivity and decreased accuracy

\section{(a)}

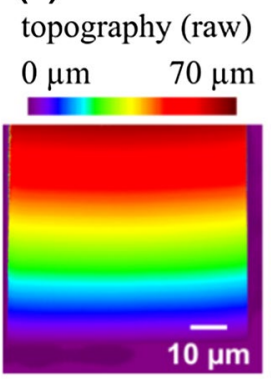

- average plane

-2D polynomial

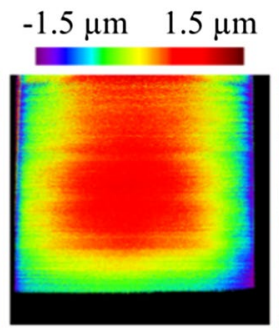

(b) optical
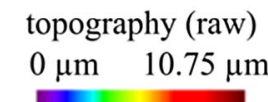

$-2 \mathrm{D}$ rot. poly.
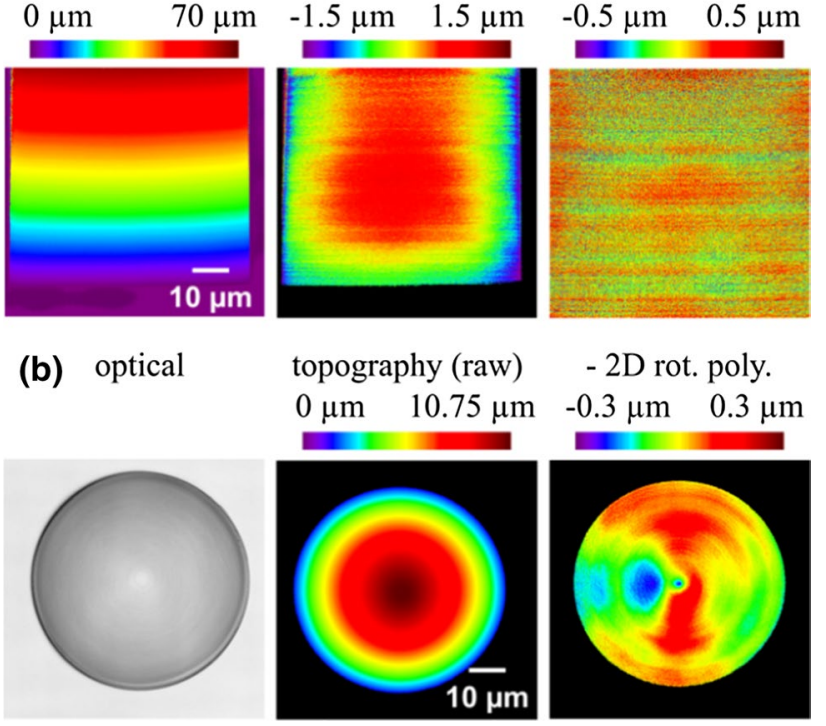

Fig. 13 Topography of fabricated TIR mirror (a) and microlenses (b)

appears very accurate (left-hand side). The average slope is $(1.033 \pm 0.001)$ instead of exactly 1 , corresponding to a $45^{\circ}$ angle in the design. The small deviation is due to the refractive index mismatch of the OC-I, leading to a small elongation of the entire structure. When a plane is subtracted from the surface of the TIR (middle image), the entire surface is slightly elevated in the center due to shrinkage of the outer regions. This shrinkage can be compensated by numerical correction of the computer design. The contour lines finally become visible after polynomial background subtraction (the right-hand side of Fig. 13). They are responsible for the remaining surface roughness of $226 \mathrm{~nm}$ (RMS). However, this roughness is only present on surfaces that are not parallel to the slicing direction. Simple cubes reveal an RMS roughness less than $15 \mathrm{~nm}$ on their top surface. The surface of the fabricated microlens appears very smooth under an optical microscope (the left-hand side of Fig. 13b). In comparison with annular hatching strategies (Malinauskas et al. 2010), no ring-shaped undulations of the surface are apparent. These are normally induced by minor laser power instabilities that impact the surface formation as $2 \mathrm{PP}$ is strongly dependent $\left(\alpha I^{2}\right)$ on the applied photon dose (Steenhusen et al. 2015). In case of galvanometric mirror exposure, these instabilities are averaged out resulting in the smooth surfaces. LSM characterization is shown in the middle of Fig. 13b. When the deviations are observed from the intended spherical profile (not shown), the center of the lens is slightly elevated and the margins are lowered. This is also due to linear stretching of the entire volume. Again, after the polynomial background is subtracted, the contour lines become visible on the surface. The resulting RMS roughness is $70 \mathrm{~nm}$, which is lower than in the case of the TIR mirror because the slicing distance was lowered from 0.25 to $0.1 \mu \mathrm{m}$. It can be further decreased by using ORMOCER ${ }^{\circledR} \mathrm{S}$ with a more distinct proximity effect that smooth individual hatching lines.

The results have been transferred to the fabrication in SLE fabricated channels as shown in Fig. 14. In that case the structures were fabricated using the NA $=0.6$ lens, because (a)

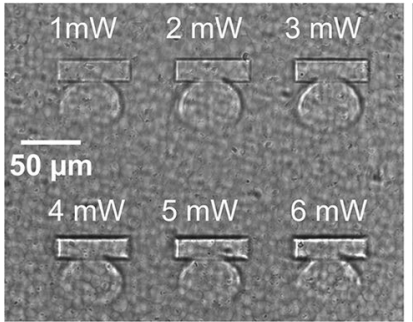

(c)

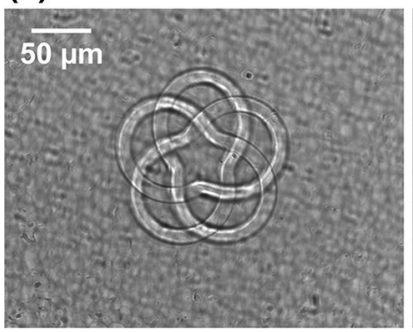

(b)

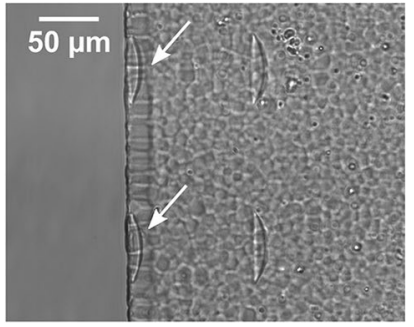

(d)

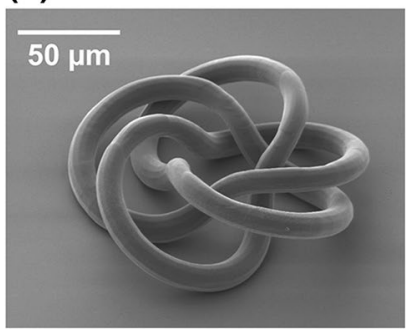

Fig. 14 Structures fabricated inside microfluidic channels (a-c). SEM image of knot structure for comparison on cover slip (d) 
the working distance of the oil-immersion objective is too low to reach the SLE channels for the available chips.

Figure 14a depicts the flap inside a large channel. Here, the laser power was varied, resulting in more polymer conversion, larger voxels and consequently more image contrast at higher powers. Figure $14 \mathrm{~b}$ clearly shows that $2 \mathrm{PP}$ structures can also be aligned directly to the walls of SLE channels (highlighted with arrows). In that picture standing microlenses were created on the walls and also inside the channel, only suspended at the top and the bottom of the channel. No severe surface deteriorations can be deduced from microscopic characterization. Neither the surface roughness of the channels nor the refractive index mismatch of fused silica and OC-I appears to impact the structure formation. However, thorough topographic characterization inside microchannels is difficult because they are not accessible (which is indeed an advantage of SLE because they do not have to be sealed after fabrication). Hence, SEM and LSM fail as imaging technologies. In the future, 3D fluorescence imaging might be an option to probe the 3D structures inside channels because OC-I reveals a noticeable fluorescence signal. Finally Fig. 14c shows a 3D knot fabricated inside a channel. For comparison, an SEM image of the counterpart fabricated on a cover slip is depicted in Fig. 14d. This demonstrates that the process described here has great potential for generating very sophisticated 3D structures.

\section{Conclusion}

In comparison with conventional polishing processes, laser polishing makes it possible to process internal walls in microchannels. With laser polishing, thin fused silica walls can be heated up with laser radiation in order to smooth the surface of the inner channel due to the surface tension. On account of remelting process, inside surfaces of microchannels can be polished and, hence, microfluidic processes and particles inside the microchannel observed due to the reduced micro roughness. In a measuring field of 1 by $1 \mathrm{~mm}^{2}$, the inner surface roughness can be reduced from $1.2 \mu \mathrm{m}$ down to $3 \mathrm{~nm}$ for spatial wavelength $\lambda<400 \mu \mathrm{m}$.

We have demonstrated that SLE and laser polishing can be combined by creating a microfluidic with microchannels $500 \mu \mathrm{m}$ below the surface and a cross-sectional area of $300 \times 300 \mu \mathrm{m}^{2}$.

Additionally, we have demonstrated that 2PP structures might enable new functions for micro- and optofluidic applications. Depending on the focusing optics, processing conditions and slicing/hatching distances, different levels of $\mu \mathrm{m}$-scale precision can be achieved with a roughness down to $70 \mathrm{~nm}$. As these structures can be manufactured in SLE channels, the processes described in this paper can unlock great potential to manufacture new devices in the future.
Open Access This article is distributed under the terms of the Creative Commons Attribution 4.0 International License (http://creativecommons.org/licenses/by/4.0/), which permits unrestricted use, distribution, and reproduction in any medium, provided you give appropriate credit to the original author(s) and the source, provide a link to the Creative Commons license, and indicate if changes were made.

\section{References}

Amato L, Gu Y, Bellini N, Eaton SM, Cerullo G, Osellame R (2012) Integrated three-dimensional filter separates nanoscale from microscale elements in a microfluidic chip. Lab Chip 12:1135-1142

Burmeister F, Steenhusen S, Houbertz R, Asche TS, Nickel J, Nolte S, Tucher N, Josten P, Obel K, Wolter H, Fessel S, Schneider AM, Gärtner K-H, Beck C, Behrens P, Tünnermann A, Walles $\mathrm{H}$ (2015) Two-photon polymerization of inorganic-organic polymers for biomedical and microoptical applications. In: König K, Ostendorf A (eds) Optically induced nanostructures-biomedical and technical applications, De Gruyter, Berlin, Boston

Cheng Y et al (2003) Three-dimensional micro-optical components embedded in photosensitive glass by a femtosecond laser. Opt Lett 28:1144-1146

Cormont $\mathrm{P}$, Combis $\mathrm{P}$, Gallais L, Hecquet $\mathrm{C}$, Lamaignère L, Rullier JL (2013) Removal of scratches on fused silica optics by using a $\mathrm{CO}_{2}$ laser. Opt Express 21:28272-28289

Doraiswamy A, Patz T, Narayan RJ, Chichkov B, Ovsianikov A, Houbertz R et al. (2005) Biocompatibility of CAD/CAM ORMOCER polymer scaffold structures. Mater Res Soc Symp. doi:10.1557/ PROC-845-AA2.4

Eaton SM, de Marco C, Martinez-Vazquez R, Ramponi R, Turri S, Cerullo $G$ et al (2012) Femtosecond laser microstructuring for polymeric lab-on-chips. J Biophotonics 5:687-702

Eaton SM, Osellame R, Ramponi R (2015) Femtosecond laser micromachining for the realization of fully integrated photonic and microfluidic devices. In: Broquin JE, Conti GN (eds) Integrated optics: devices, materials, and technologies XIX, vol 9365. San Francisco, CA, USA

Fanderlik I (1991) Silica glass and its application. New York. Elsevier, Amsterdam

Fessel S, Schneider A, Steenhusen S, Houbertz R, Behrens P (2012) Towards an atomistic model for ORMOCER ${ }^{\circledR}$-I: application of forcefield methods. J Sol Gel Sci Technol 63:356-365

Focsan M, Craciun AM, Astilean S, Baldeck PL (2016) Two-photon fabrication of three-dimensional silver microstructures in microfluidic channels for volumetric surface-enhanced Raman scattering detection. Opt Mater Express 6:1587-1593

He F, Lin J, Cheng Y (2011) Fabrication of hollow optical waveguides in fused silica by three-dimensional femtosecond laser micromachining. Appl Phys B 105:379-384

Heidrich S, Willenborg E, Weingarten C, Temmler A (2015) Laser polishing and laser form correction of fused silica optics. Mat.wiss. u. Werkstofftech 46:668-674

Heptonstall A et al. (2014) Enhanced characteristics of fused silica fibers using laser polishing. Class Quantum Grav 31(10): 105006. doi:10.1088/0264-9381/31/10/105006

Hermans M (2014) Selective, laser-induced etching of fused silica at high scan-speeds using KOH. JLMN 9:126-131

Hermans M, Gottmann J, Schiffer A (2012) In-situ diagnostics on fs-laser induced modification of glasses for selective etching. In Bachmann FG, Pfleging W, Washio K, Amako J, Hoving W, Lu Y (eds) SPIE LASE. SPIE, 82440E

Hildebrand J, Hecht K, Bliedtner J, Müller H (2011) Laser beam polishing of quartz glass surfaces. Phys Procedia 12:452-461 
Houbertz R, Steenhusen S, Stichel T, Sext G (2010) Two-photon polymerization of inorganic-organic hybrid polymers as scalable technology using ultra-short laser pulses. In: Duarte FJ (ed) Coherence and ultrashort pulse laser emission, InTech, pp 583-608

Iosin M, Scheul T, Nizak C, Stephan O, Astilean S, Baldeck P (2011) Laser microstructuration of three-dimensional enzyme reactors in microfluidic channels. Microfluid Nanofluid 10:685-690

Jung S, Lee PA, Kim BH (2016) Surface polishing of quartz-based microfluidic channels using $\mathrm{CO}_{2}$ laser. Microfluid Nanofluid 20(6):1-6. doi:10.1007/s10404-016-1748-8

Lin C-L, Liu Y-J, Lin Z-D, Wu B-L, Lee Y-H, Shin C-S et al (2015) Laser direct writing 3D structures for microfluidic channels: flow meter and mixer. Proc SPIE. doi:10.1117/12.2079547

Malinauskas M, Gilbergs H, Zukauskas A, Purlys V, Paipulas D, Gadonas R (2010) A femtosecond laser-induced two-photon photopolymerization technique for structuring microlenses. J Opt 12:035204. doi:10.1088/2040-8978/12/3/035204

McLachlan Anthony D, Meyer Fred P (1987) Temperature dependence of the extinction coefficient of fused silica for $\mathrm{CO}_{2}$ laser wavelengths. Appl Opt 9:1728-1731

Nowak KM, Baker HJ, Hall DR (2006) Efficient laser polishing of silica micro-optic components. Appl Opt 45:162

Sanchez C, Belleville P, Popall M, Nicole L (2011) Applications of advanced hybrid organic-inorganic nanomaterials: from laboratory to market. Chem Soc Rev 40:696-753

Serhatlioglu M, Ortaç B, Elbuken C, Biyikli N, Solmaz ME (2016) $\mathrm{CO} 2$ laser polishing of microfluidic channels fabricated by femtosecond laser assisted carving. J Micromech Microeng 26:115011

Felix Sima, Dong Wu, Jian Xu, Katsumi Midorikawa, Koji Sugioka (2015) Ship-in-a-bottle integration by hybrid femtosecond laser technology for fabrication of true 3D biochips. Proc SPIE. doi:10.1117/12.2081343

Steenhusen S, Stichel T, Houbertz R, Sextl G (2010) Multi-photon polymerization of inorganic-organic hybrid polymers using visible or IR ultrafast laser pulses for optical or optoelectronic devices. Proc SPIE 7591:759114

Steenhusen S, Burmeister F, Eckstein H-C, Houbertz R (2015) Twophoton polymerization of hybrid polymers for applications in micro-optics. Proc SPIE. doi:10.1117/12.2079277
Stichel T, Hecht B, Houbertz R, Sextl G (2010) Two-photon polymerization as method for the fabrication of large scale biomedical scaffold applications. J Laser Micro Nanoeng 5:209-212

Sugioka K, Cheng Y (2014a) Fabrication of 3D microfluidic structures inside glass by femtosecond laser micromachining. Appl Phys A 114:215-221

Sugioka K, Cheng Y (2014) Femtosecond laser three-dimensional micro- and nanofabrication. Appl Phys Rev 1:041303. doi: $10.1063 / 1.4904320$

Waheed S, Cabot JM, Macdonald NP, Lewis T, Guijt RM, Paull B et al (2016) 3D printed microfluidic devices: enablers and barriers. Lab Chip 16:1993-2013

Weingarten C, Heidrich S, Wu Y, Willenborg E (2015) Laser polishing of glass. In: SPIE Optifab. International society for optics and photonics, vol 9633

Weingarten C, Schmickler A, Willenborg E, Wissenbach K, Poprawe R (2017) Laser polishing and laser shape correction of optical glass. J Laser Appl 29:11702. doi:10.2351/1.4974905

Wu D, Wu S-Z, Xu J, Niu L-G, Midorikawa K, Sugioka K (2014) Hybrid femtosecond laser microfabrication to achieve true 3D glass/polymer composite biochips with multiscale features and high performance: the concept of ship-in-a-bottle biochip. Laser Photonics Rev 8:458-467

Wu D, Xu J, Niu L-G, Wu S-Z, Midorikawa K, Sugioka K (2015) In-channel integration of designable microoptical devices using flat scaffold-supported femtosecond-laser microfabrication for coupling-free optofluidic cell counting. Light Sci Appl 4:e228. doi:10.1038/lsa.2015.1

Wu D, Xu J, Niu L-G, Wu S-Z, Midorikawa K, Sugioka K (2015b) In-channel integration of designable microoptical devices using flat scaffold-supported femtosecond-laser microfabrication for coupling-free optofluidic cell counting. Light Sci Appl 4:e228

Xiong W, Zhou YS, He XN, Gao Y, Mahjouri-Samani M, Jiang L et al. (2012) Simultaneous additive and subtractive three-dimensional nanofabrication using integrated two-photon polymerization and multiphoton ablation. Light Sci Appl 1:e6. doi:10.1038/1sa.2012.6 